\title{
Influencia de la televisión y videojuegos en el aprendizaje y conducta infanto-juvenil
}

\author{
VALERIA ROJAS O. ${ }^{1}$ \\ 1. Pediatra, Neuróloga Infantil. Presidenta Comité de Medios y Salud Infanto-Juvenil Sociedad Chilena de Pediatría.
}

Palabras clave: Aprendizaje, trastornos, televisión, tv, videojuegos, desarrollo.

\section{Introducción}

En las últimas décadas, junto con el explosivo desarrollo tecnológico, especialmente de las llamadas Tecnologías de la Información y Comunicación (TIC) que incluyen televisión (TV), radio, música, videojuegos, telefonía, Internet, etc; se ha comprobado también, la gran influencia que estas tecnologías, a través de su omnipresencia en los hogares, escuelas, instituciones, etc, tienen sobre la salud infanto-juvenil. Nuevas investigaciones, han dado cuenta de dicha influencia, particularmente en aspectos como conducta violenta, consumo de alcohol y drogas, conductas sexuales de riesgo, trastornos alimentarios, nutritivos y otros ${ }^{1,2}$. Muchas de estas investigaciones han comenzado a dar luces sobre las bases neurológicas y neuropsicológicas de este fenómeno y las autoridades de salud de muchos países desarrollados han implementado políticas y medidas regulatorias y de educación para los niños y sus familias ${ }^{3}$.

Existe actualmente conciencia de que los pediatras deben dialogar respecto a la exposición a estas tecnologías con los niños y sus padres y entregar educación basada en investigaciones científicas respecto al uso apropiado de los medios.
En Chile, este problema de Salud Pública no está suficientemente asumido y no se le ha dado aún la atención que se merece.

El objetivo de esta revisión es explorar las evidencias actuales de los efectos de la televisión y videojuegos en el consumo, aprendizaje y conducta de niños y jóvenes y entregar recomendaciones a los pediatras y profesionales de la salud que trabajen con niños, para orientar a los pacientes, sus familias y la comunidad en general en el uso apropiado de las TIC.

\section{Tiempo de exposición a TV}

En Chile, la encuesta AdimarK-Time Ibope y Mindshare (2007) reveló que los niños de 4 a 12 años ven 4 horas de TV por día, el 70\% de niños menores de 3 años ve TV en forma habitual y el $75 \%$ de niños tiene TV en su pieza.

En la Sexta Encuesta Nacional del Consejo Nacional de Televisión (2008) hay 2,4 televisores por hogar y $53 \%$ de ellos posee computador. Un 57,7\% dijo haber visto contenidos inadecuados tales como vocabulario grosero, escenas de sexo, violencia, trato discriminatorio y contenidos de adultos en horario para menores.

Además del tiempo frente a la pantalla hay otros factores que influencian el efecto de los medios en niños incluyendo el nivel de desarrollo, susceptibilidad individual y si estos ven TV solos o acompañado de sus padres. 


\section{Desarrollo cerebral, $T V$ y videojuegos}

La televisión es una ventana al mundo que les permite a los niños desarrollar una gran sensibilidad y conocimiento, como por ejemplo por la ecología y el mundo animal; es parte integral de nuestra sociedad, y no solamente es informativa sino que también entretenida y permite además entregar educación ya sea directa o indirectamente en diversos aéreas incluyendo temas de salud pública. Los videojuegos y la TV son una entretención "segura", sin riesgo de accidente y permite a los padres realizar otras labores con tranquilidad ${ }^{4}$.

Sin embargo, es necesario tener en cuenta que el desarrollo neurológico y emocional del niño es distinto del adulto. La maduración cerebral finaliza alrededor de los 18 a 20 años y este proceso a nivel cortical ocurre desde aéreas posteriores hacia anterior, es decir, las áreas prefrontales son la ultimas en madurar. La corteza prefrontal (CPF) juega un papel crucial en funciones cerebrales superiores como la cognición, el control de la conducta, reflexión, juicio, etc $^{5}$.

En niños menores de 5 años existe una marcada capacidad de fantasía y perciben las imágenes de la TV como reales y verdaderas. A diferencia del adulto, antes de los 10 años la capacidad de razonamiento crítico y relacional está limitado. Por lo anterior los menores de 10 años no deberían estar expuestos a telenovelas ni noticieros de TV cuyo sensacionalismo y crudeza de imágenes pueden provocar trastornos del sueño y miedos que alteran su diario vivir. Como alternativa podrían acceder a noticias a través de medios escritos que tienen un impacto emocional menor que la "pantalla".

La adolescencia temprana es una etapa de desorganización de la personalidad y de inestabilidad de las conductas. En el púber la dinámica central es encontrarse a si mismo y autodefinir la identidad. Los programas actuales dedicados a adolescentes, en general, carecen de contenido valórico y no responden a las necesidades e interrogantes propias de esta edad. Investigaciones han demostrado que adolescentes expuestos a mayor contenido sexual en los medios tienen más probabilidades de iniciar actividad sexual a más temprana edad ${ }^{6-9}$.

\section{Desarrollo emocional y marketing}

En la infancia los comerciales tienen una gran influencia en promover el consumismo infantil y en generar en los padres presión por comprar determinadas marcas. Investigaciones han demostrado que niños pequeños, menores de 8 , están cognitiva y psicológicamente indefensos frente a la publicidad ${ }^{2}$. Muchos niños preescolares no entienden la diferencia entre un programa creado para entretener y un comercial creado para vender. En poblaciones económicamente vulnerables la exposición a comerciales genera frustración y violencia ${ }^{10,11}$.

Una publicación de la Academia Americana de Pediatría del año 2001 reveló que los niños ven más de 20.000 comerciales cada año ${ }^{12}$. Un estudio chileno realizado en 469 niñ@s previo al Día del Niño, en estratos medio alto y medio bajo, $61 \%$ de los menores de 7 años pidió de regalo un producto que vio en TV. De ellos hubo diferencias entre quienes poseían un aparato de TV en su pieza $(55 \%)$ en relación a quienes no tenían equipo en su habitación $(28 \%)^{13}$.

Frente a esta evidencia algunos países como Suecia y Noruega han prohibido toda publicidad dirigida a menores de 12 años, Grecia permite publicidad de juguetes sólo después de las 22 horas. Dinamarca y Bélgica restringen severamente la publicidad dirigida a los niños ${ }^{11}$.

\section{$T V$ videojuegos y aprendizaje}

La televisión y particularmente los videojuegos pueden ser una poderosa herramienta educativa, especialmente los videojuegos ya que poseen tres características especiales. En primer lugar el niño no es solo un espectador, si no que interactúa y se transforma en el actor principal del juego. Por otro lado, consigue la atención absoluta del jugador y tiene un refuerzo positivo a la acción ejecutada en forma inmediata. Debido a lo anterior si el contenido del juego es violento, la conducta violenta será aprendida fácilmente ${ }^{14,15}$.

Programas como Plaza Sésamo han sido un ejemplo de cómo preescolares pueden aprender valores y conductas positivas sobre diversidad racial y cultural, tolerancia, cooperación, amabilidad, aritmética simple y vocabulario a través de un formato televisivo entretenido ${ }^{16}$. 
En Chile, NOVASUR ha sido pionero en crear Programas Educativos para televisión que son accesibles a todas escuelas públicas. (http// www.novasur.cl). Sin embargo, en nuestro país algunos programas de televisión abierta, orientados a niños sobre el mundo animal, son emitidos en horarios no apropiados para ellos (22:00 $\mathrm{hr}$ ) siendo reconocido que los niños entre 4 y 12 años deben dormir 10 horas diarias, de tal manera de no alterar el ciclo sueno-vigilia.

La TV, videojuegos, etc, desplazan el tiempo dedicado a lectura y tareas escolares ${ }^{17}$. Muchos estudios bien controlados han mostrado que aún 1 ó 2 horas por día de TV no supervisada en niños escolares provoca un significativo efecto deletéreo en rendimiento académico, especialmente en lectura ${ }^{18,19}$. En un reciente estudio de la Universidad alemana de Würzburg, tras examinar durante cuatro años a más de 350 niños de 4 a 8 años de edad con diversos consumos de TV (alto: 117 minutos diarios, medio: 69 minutos diarios y bajo 35 minutos diarios), los investigadores concluyeron que el exceso de televisión entretenida tiene una clara y negativa influencia en la capacidad de los pequeños a la hora de comprender lo que leen y, por ende, en su rendimiento acadé$\operatorname{mico}^{20}$.

Otros investigadores han encontrado que los entretenimientos electrónicos están llevando a una perturbadora declinación en la capacidad de juegos imaginativos entre los niños. Niños que tienen más capacidad imaginativa, desarrollan mayor destreza en su vocabulario en relación a niños que dedican menos tiempo a juegos creativos. El desarrollar precozmente estas destrezas, predice la habilidad para ser creativo y generar alternativas de soluciones en los problemas de la vida diaria ${ }^{21,22}$.

\section{Televisión videojuegos y conducta violenta}

Según la teoría Cognitiva Social de Albert Bandura el ambiente, en el proceso de maduración cerebral, modela la conducta. El investigador demostró en numerosos estudios experimentales que los niños que observan conductas agresivas recompensadas suelen repetirlas posteriormente con mayor frecuencia que aquellos que observan esa misma conducta castigada ${ }^{23}$. Con el desarrollo de la medicina ha sido posible observar in vivo a través de la Resonancia Nuclear Magnética Funcional la potente activación de áreas relacionadas con la conducta violenta, como la amígdala y el cortex cingulado anterior dorsal, mientras se juegan videojuegos violentos ${ }^{24,25}$.

El psicólogo Jeffrey Johnson, del New York Psychiatric Center, publicó en Science el año 2002 un estudio de 707 casos, analizados a lo largo de 17 años, a partir de 1975. Se trataba de establecer una relación entre el tiempo dedicado a ver televisión y las conductas agresivas de los individuos observados. El proyecto consideraba un seguimiento a la conducta como televidentes de las familias y la revisión de los datos de los archivos del Estado de New York y de la Oficina Federal de Investigaciones (FBI) que registraban las detenciones y acusaciones de conducta criminal en los años del estudio. El resultado de la investigación reportó una relación significativa entre la cantidad de tiempo que se pasó viendo televisión durante la adolescencia y el inicio de la edad adulta, y la probabilidad de actos agresivos posteriores contra terceros, relación que se mantuvo incluso después de controladas variables como la conducta agresiva anterior, el abandono infantil, el ingreso familiar, la violencia en el barrio la educación de los padres y afecciones psiquiátricas $^{26}$.

La observación e imitación en los niños pequeños toman como modelo a los padres, educadores, amigos y aquello que observan a través de los medios. En la televisión abierta de nuestro país el $89 \%$ de las caricaturas en el horario infantil tienen escenas de violencia, ya sea física, psicológica o verbal ${ }^{27,28}$.

En un estudio prospectivo de factores predictores de Bullying en 12666 niños en edad escolar, se demostró que una precoz estimulación cognitiva (ej: paseos, lectura, juegos, rol parental en la enseñanza del niño) y apoyo emocional (ej: comidas con padres, padres llaman a hijos mientras trabajan) como factores protectores, y la exposición a TV como factor de riesgo, fueron cada una e independientemente asociadas con conductas matonescas en edad esco$\operatorname{lar}^{29}$.

En un artículo de revisión del Pediatrics del año 2001 en que participaron la Academia 
Americana de Pediatría, la Asociación Médica Americana, la Academia Americana de Psiquiatría de la Infancia y Adolescencia y la Asociación Americana de Psicología, expusieron las siguientes conclusiones respecto a los tres efectos principales que la violencia en los medios tiene en el público ${ }^{30}$.

1) El efecto mimético directo: niños y adultos expuestos a grandes dosis de espectáculos violentos pueden llegar a ser más agresivos o a desarrollar, con el tiempo, actitudes favorables al uso de la violencia como medio para resolver los conflictos.

Los niños aprenden por observación y probando patrones conductuales. Repetida exposición a patrones conductuales violentos puede llevar a aumentar sentimientos de hostilidad, desensibilización al dolor de otros, aumento de la probabilidad de interactuar y responder a otros con violencia. Los videojuegos violentos son un ambiente ideal para aprender a ser violento lo que explicaría el 13\%-22\% del aumento de la conducta violenta de los jóvenes.

2) El segundo es un efecto más indirecto: la insensibilización. Los espectadores, sobre todo los niños, expuestos a grandes cantidades de violencia en la pantalla, pueden hacerse menos sensibles a la violencia real del mundo que les circunda, menos sensibles al sufrimiento ajeno, y más predispuestos a tolerar el aumento de violencia en la vida social. La violencia en sí misma no es el problema, sino como ésta es retratada, esto hace la diferencia entre aprender acerca de la violencia y aprender a ser violento. Estimulando la violencia en un contexto sexual o cómico es particularmente peligroso, porque se asocian sentimientos positivos con el dañar a otros.

3) El público puede sobreestimar el índice de violencia real y creer que la sociedad en la que vive se caracteriza por un elevado grado de violencia y peligrosidad. En algunos niños la exposición a la violencia mediática los lleva a generar ansiedad, depresión, trastornos del sueño, pesadillas, estrés post traumático, etc ${ }^{31}$.

\section{Recomendaciones para los médicos y profesionales de la salud}

Los profesionales de la salud deben permanecer conscientes y alertas acerca de los efec- tos de la TV y videojuegos en los niños, incluyendo conducta agresiva y violenta, obesidad, pobre autoimagen, uso de sustancias, trivialización del sexo y la sexualidad, actividad sexual precoz, incremento de la pasividad y abulia, pérdida de tiempo y de la posibilidad de aprender y ejercitar conductas sociales positivas. El interrogatorio acerca de los hábitos en relación a los medios, tiempo frente a TV, uso de videojuegos y computador, especialmente en niños de conducta agresiva y de familias vulnerables, debería ser habitual en la consulta y controles de salud ${ }^{32}$.

Es necesario estar familiarizado con los diferentes tipos de tecnologías a los cuales los pacientes están expuestos.

Se debe crear conciencia precozmente en la vida del niño y su familia sobre los efectos de $\mathrm{TV}$, videojuegos y otras TIC y establecer claramente las reglas y el buen uso de estas tecnologías tal como sigue:

- Se debe promover que la exposición a medios sea en familia y discutir su contenido. En los niños se debe promover un análisis crítico respecto a lo que ven. Comentar con los niños sobre la violencia y el sufrimiento que esta provoca.

- Los padres deben ayudar a los niños a diferenciar realidad de fantasía, especialmente en relación al sexo, violencia y comerciales.

- El tiempo de pantalla debe ser limitado a 1,5 horas los días de clases y a 2 horas los fines de semana y vacaciones. No encender la TV al azar.

- Una hora antes de dormir sin TV ni PC (promover la higiene del sueño): la falta de sueño provoca al día siguiente distractibilidad e irritabilidad.

- No se debe permitir que niños tengan TV, PC o Consolas de videojuegos en sus dormitorios. Especialmente Internet debe estar en un lugar de paso, y las claves de acceso deben ser comunes a toda la familia.

- Enseñar a padres a chequear, previo al uso o compra de un videojuego, las edades para el cual está permitido, a través del sello que traen en las cajas (figura 1) o través de página web: www. esrb.org

- En los niños mayores promover la planificación de los programas a ver y sus horarios. 


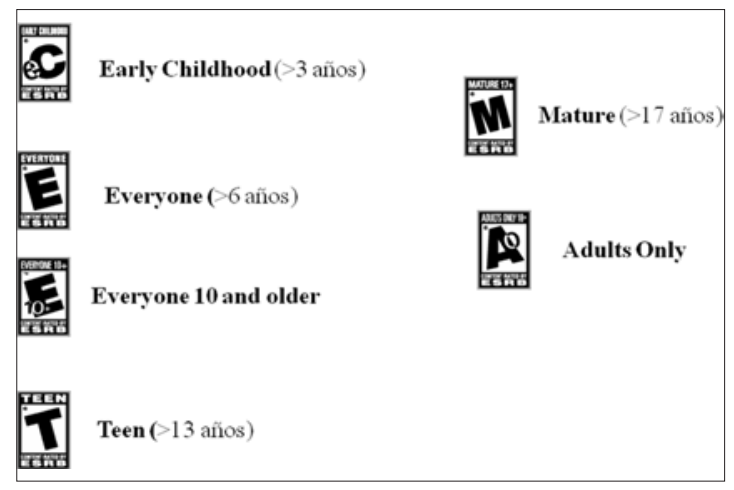

Figura 1.

Sin embargo, idealmente esto debe realizarse bajo las supervisión de los padres y estos deben explicar porque algunos programas no son apropiados para ellos.

- Los padres deben ser modelos positivos en el consumo de los medios de tal manera que cuando los imiten obtengan resultados satisfactorios y alentadores.

- Los niños menores de 2 años no deben ver TV. Explicar que es la etapa del mayor desarrollo psicomotor y el tiempo frente a la pantalla es un tiempo perdido en este importante proceso.

- Alentar alternativas de entretenimientos para niños, incluyendo lectura, deportes, hobbies y juegos creativos.

\section{Conclusiones}

Las Tecnologías de la Información y Comunicación han transformado la vida cotidiana de los niños y sus familias. Pertenecemos a una cultura que no puede entenderse ni existe al margen de los medios de comunicación. Es necesario por lo tanto "preparar" al niño a comprender la cultura mediática, a tener un análisis crítico y a participar en ella propositivamente. La incorporación de un currículo de Educación en Medios en el sistema educativo, en los distintos niveles y bajo diversas modalidades, sería un paso muy importante para avanzar en esta preparación.

Es necesario también que se establezcan ciertas normas mínimas de carácter ético para la publicidad; hay que impulsar políticas, planes y programas relativos al rol de la familia ante los medios, al mismo tiempo que formular políticas públicas que garanticen la calidad de los programas dirigidos a los niños.

Como pediatras y trabajadores de la salud debemos involucrarnos en promover cambios educacionales y legales que aseguren el sano desarrollo físico y mental de nuestros niños y adolescentes.

\section{Referencias}

1.- American Academy of Pediatrics, Committee on Communications: Children, adolescents, and television. Pediatrics 1995; 96: 786-7.

2.- Canadian Paediatric Society, Psychosocial Paediatrics Committee: Impact of media use on children and youth. Paediatrics \& Child Health 2003; 8: 301-6.

3.- Luke C: Television and Your Child-A Guide for Concerned Parents. Toronto Ontario, Kagan and Woo, Ltd. 1988

4.- Wright JC, Auston A, et al: Effects of educational television viewing of lower income preschoolers on academic skills, school readiness and school adjustment one to three years later. Child Development 2001; 72: 1347-66.

5.- Lenroot RK, Giedd JN: Brain development in children and adolescents: insights from anatomical magnetic resonance imaging. Neurosci Biobehav Rev 2006; 30: 718-29.

6.- Giedd JN: Structural magnetic resonance imaging of the adolescent brain. Ann N Y Acad Sci 2004; 1021: 77-85.

7.- Giedd JN: The teen brain: insights from neuroimaging J Adolesc Health 2008; 42: 335-43. Review.

8.- Collins RL, Elliott MN, Berry SH, Kanouse DE, Kunkel D, Hunter $S B$, et al: Watching sex on television predicts adolescent initiation of sexual behavior. Pediatrics 2004; 114: e280-9.

9.- Brown JD, L'Engle KL, Pardun CJ, Guo G, Kenneavy K, Jackson C: Sexy media matter: exposure to sexual content in music, movies, television, and magazines predicts black and white adolescents' sexual behavior. Pediatrics 2006; 117: 1018-27.

10.- American Academy of Pediatrics: Committee on Communications Children, Adolescents, and Advertising, Pediatrics 2006; 118: 2563-9.

11.- Chamberlain LJ, Wang Y, Robinson TN: Does children's screen time predict requests for advertised products? Cross-sectional and prospective analyses. Arch Pediatr Adolesc Med 2006; 160: 363-8.

12.- American Academy of Pediatrics, Committee on Communications: Children, adolescents, and advertising. Pediatrics 2001; 107: 423-6.

13.- Uribe R, Hidalgo $V$ : La influencia de la Publicidad Televisiva en los niños: ¿Qué sabemos del tema en Chile?. Revista de Economía y Administración, Mayo/ 
Junio 2006, No 152 pag 22-8.

14.- Gentile DA, Lynch PJ, Linder JR, Walsh DA: The effects of violent video game habits on adolescent aggressive attitudes and behaviors. Journal of Adolescence 2004; 27: 5-22.

15.- Anderson $C A$. An update on the effects of violent video games. Journal of Adolescence 2004; 27: 11322.

16.- Huston AC, Anderson DR, Wright JC, Linebarger D, Schmitt KL: Sesame Street viewers as adolescents: The Recontact study. In: Fisch S, Truglio R, eds. G is for growing: Thirty Years of Research on Sesame Street. Mahwah, NJ: Erlbaum, 2000.

17.- Strasburger VC: Does television affect learning and school performance? Pediatrician 1986; 38: 141-7.

18.- Thakkar RR, Garrison MM, Christakis DA: A systematic review for the effects of television viewing by infants and preschoolers. Pediatrics 2006; 118 (5): 2025-31.

19.- Barr-Anderson DJ, van den Berg P, Neumark-Sztainer $D$, Story M: Characteristics associated with older adolescents who have a television in their bedrooms. Pediatrics 2008; 121: 718-24.

20.- Ennemoser M, Schneider $W$ : Relations of Television Viewing and Reading: Findings From a 4-Year Longitudinal Study. Journal of Educational Psychology 2007; 99: 349-68.

21.- Rice ML, Woodsmall L: Lessons from television: Children's word learning when viewing. Child Dev 1988; 59: 420-9.

22.- Hancox RJ, Milne BJ, Poulton R: Association of Television Viewing During Childhood With Poor Educational Achievement. Arch Pediatr Adolesc Med 2005; 159: 614-8.

23.- Bandura $A$, et al: Imitation of Film-Mediated Aggressive Model. Journal of Abnormal and Social
Psychology, 1963.

24.- Mathiak K, Weber R: Toward brain correlates of natural behavior: fMRI during violent video games. Hum Brain Mapp 2006; 27: 948-56.

25.- Anderson CA, Bushman BJ: Effects of violent games on aggressive behavior, aggressive cognition, aggressive affect, physiological arousal, and prosocial behavior: A meta-analytic review of the scientific literature. Psychological Science 2001; 12: 353-9.

26.- Johnson JG, Cohen P, Smailes EM, Kasen S, Brook $J S$ : Television viewing and aggressive behavior during adolescence and adulthood. Science 2002; 295 (5564): 2468-71.

27.- Carrasco S. Consejo Nacional de Televisión-Chile. Revista CNTV - TV y Violencia (Diciembre-2002). Violencia en TV, el gran Debate Barómetro pag 20-25.

28.- Comstock GA, Paik H: The effects of television violence on antisocial behavior: A meta-analysis. Communication Research 1994; 21: 516-46.

29.- Zimmerman F, Glew G, Christakis D, Katon W: Early Cognitive Stimulation, Emotional Support, and Television Watching as Predictors of Subsequent Bullying Among Grade-School Children. Arch Pediatr Adolesc Med 2005; 159: 384-8.

30.- American Academy of Pediatrics. Media violence. Pediatrics 2001; 108: 1222-6.

31.- Otto MW, Henin A, Hirshfeld-Becker DR, Pollack MH, Biederman J, Rosenbaum JF: Posttraumatic stress disorder symptoms following media exposure to tragic events: Impact of 9/11 on children at risk for anxiety disorders. J Anxiety Disord 2007; 21 (7): 888-902.

32.- Robinson TN, Wilde ML, Navracruz LC, Haydel KF, Varady A: Effects of reducing children's television and video game use on aggressive behavior: a randomized controlled trial. Arch Pediatr Adolesc Med $2001 ; 155$ (1): 17-23. 